\title{
A Priori “Imprinting” of General Relativity Itself on Some Tests of It?
}

\author{
Lorenzo Iorio \\ INFN-Sezione di Pisa, Viale Unità di Italia 68, 70125 Bari, Italy \\ Correspondence should be addressed to Lorenzo Iorio, lorenzo.iorio@libero.it
}

Received 14 January 2010; Accepted 23 February 2010

Academic Editor: Zeljko Ivezic

Copyright () 2010 Lorenzo Iorio. This is an open access article distributed under the Creative Commons Attribution License, which permits unrestricted use, distribution, and reproduction in any medium, provided the original work is properly cited.

We investigate the effect of possible a priori "imprinting" effects of general relativity itself on satellite/spacecraft-based tests of it. We deal with some performed or proposed time-delay ranging experiments in the sun's gravitational field. It turns out that the "imprint" of general relativity on the Astronomical Unit and the solar gravitational constant $G M_{\odot}$, not solved for in the so far performed spacecraft-based time-delay tests, induces an a priori bias of the order of $10^{-6}$ in typical solar system ranging experiments aimed to measure the space curvature PPN parameter $\gamma$. It is too small by one order of magnitude to be of concern for the performed Cassini experiment, but it would affect future planned or proposed tests aiming to reach a $10^{-7}-10^{-9}$ accuracy in determining $\gamma$.

\section{Introduction}

Several space-based tests of the Einstein's General Theory of Relativity (GTR) have been performed, or attempted, in the more or less recent past by following an "opportunistic" approach, that is, by suitably analyzing existing data sets of artificial satellites or interplanetary spacecrafts almost always built and launched for different original purposes (e.g., satellite geodesy, geodynamics, planetology, etc.). A cornerstone result was the Cassini radio science experiment [1] which led to constraining the deviation of the PPN parameter $\gamma$ from its general relativistic value of unity at a $10^{-5}$ level $[2,3]$ through the measurement of the general relativistic time delay affecting the electromagnetic waves linking the Earth and the Cassini spacecraft during its journey to Saturn when they were in superior conjunction, that is, aligned and on opposite sides of the Sun. Many other high-precision space-based tests, aiming to reach accuracy levels as high as $10^{-7}-10^{-9}$ in determining $\gamma$, have been proposed [4-8]. Indeed, some theoretical scenarios predict deviations from GTR at such a level [9-12]; for a recent overview see, for example, [13].

In this paper we wish to critically discuss certain subtle issues pertaining the consistency of such data analyses interpreted as genuine tests of GTR. In Section 2 we outline the problem in general terms. We will move to concrete examples in Section 3 in which we will deal with the Cassinilike ranging experiments (Section 3.1). Section 4 is devoted to the conclusions.

\section{The Issue of the a Priori "Imprinting" of GTR Itself in Tests Dedicated to It: General Considerations}

In general, in such tests huge data sets from man-made interplanetary probes are confronted with the corresponding predictions computed from given dynamical models of their motion in which the relativistic effect to be tested was explicitly included, with one or more solve-for parameters $\{P\}$ accounting for it to be estimated in a least-square fashion along with many other ones $\{K\}$, not directly pertaining GTR.

Of crucial importance for interpreting such data analyses as genuine tests of GTR is to clarify how the numerical values of the models' parameters $\{F\}$ which have been kept fixed to certain reference values, that is, which have not been solved-for, have been originally obtained. From the point of view of testing GTR, it is not enough that the resulting postfit residuals of a certain directly observable quantity are 
statistically compatible with zero at a good level; the standard data reduction procedure used for the original goals of the missions exploited for the GTR test considered may not be valid, in principle, for performing a truly unbiased, genuine check of GTR which is not a "tautology".

Indeed, if the primary task of a space-based mission is, for example, to reach a certain astronomical target with a given accuracy, the only thing that is important to this aim is that the dynamical models adopted to predict the probe's motion are accurate enough; this is usually quantitatively judged by inspecting the postfit residuals of some directly measurable quantities like, for example, ranges or rangerates. How the parameters $\{F\}$ entering the models have been obtained, that is, their a priori values, does not matter at all; the only important thing is that the resulting fit of an existing set of observations is good enough to minimize the observable residuals.

Such an approach may, in principle, not be entirely adequate when the goal of the data analysis is testing a gravitational theory like GTR in an unambiguous, unbiased, and self-consistent way. In this case, the way the fixed parameters $\{F\}$ of the models have been obtained does, in fact, matter. Indeed, if one or more of them $\{I\}$ were previously obtained from different data of different bodies in such a way that they somehow retain a nonnegligible a priori "imprint" of the same effect we are now interested in, their use may bias the current test just towards the desired outcome yielding, for example, a very high accuracy corroboration. In this case, it would be more correct to use, if possible, values of such "imprinted" parameters $\{I\}$ which have been obtained independently of the effect itself whose existence we are just testing in the present data analysis, even if the accuracy of such different values of the "suspect" parameters $\{I\}$ was worse. Alternatively, if, for some reasons, such "unbiased" values are not available, $\{I\}$ should be included, if possible, in the list of the solvedfor parameters along with the one $(s)\{P\}$ accounting for the effect to be tested, and the resulting covariance matrix should be checked to inspect the correlations among them. The price to be paid may be an overall accuracy of the test not so high as that previously obtained, but we would have more epistemologically consistent, reliable, and trustable tests (inprinciple, certain positive corroborations may have theirstatus changed).

\section{Application to Some Concrete Cases}

3.1. The Cassini Radio Science Test and Other Proposed High-Accuracy Space-Based Measurements of $\gamma$. To be more definite, let us look at the Cassini radio science test. In that case, the radiotechnical data of the spacecraft traveling to Saturn were contrasted with a set of dynamical models by JPL of its motion and electromagnetic waves propagation in such a way that a correction $\Delta \gamma$ to the GTR-predicted value of the PPN parameter $\gamma$ was solved for, among other parameters, obtaining [2]

$$
\Delta \gamma \equiv|\gamma-1|=(2.1 \pm 2.3) \times 10^{-5}
$$

other authors got [3]

$$
\Delta \gamma \equiv|\gamma-1|=(-1.3 \pm 5.2) \times 10^{-5}
$$

Now, a physical parameter which is, of course, crucial in such a test is the gravitational parameter $G M_{\odot}$ of the Sun, which is the source of the relativistic time delay put on the test. It was not estimated $[2,3]$, so that its numerical value was kept fixed to the standard reference figure of the JPL DE ephemerides used. It does, in principle, contain an a priori "imprinting" by GTR itself through the same effect itself that was just tested with Cassini, in particular by $\gamma$ itself. Indeed, the numerical value of $G M_{\odot}$ comes from the fixed value of the defining Gaussian constant (see on the WEB http://ssd.jpl.nasa.gov/?constants.)

$$
k=0.01720209895 \mathrm{au}^{3 / 2} \mathrm{~d}^{-1},
$$

and from the value of the Astronomical Unit, not estimated in the Cassini tests,

$$
\mathrm{AU}=1.49597870691 \times 10^{11}( \pm 3) \mathrm{m}
$$

through

$$
\begin{aligned}
G M_{\odot} & =k^{2} \mathrm{AU}^{3} \mathrm{~d}^{-2} \\
& =1.32712440018 \times 10^{20}\left( \pm 8 \times 10^{9}\right) \mathrm{m}^{3} \mathrm{~s}^{-2}
\end{aligned}
$$

(Here we will use au for the symbol of the Astronomical Unit, like $\mathrm{m}$ for the meter, while AU will denote its numerical value in m.) AU was, in fact, obtained just through a combination of radar ranging of Mercury, Venus, and Mars, laser ranging of the moon (making use of light reflectors left on the lunar surface by Apollo astronauts), and timing of signals returned from spacecrafts as they orbit or make close passes and objects in the solar system [14]; thus, it is affected in a nonnegligible way, given the level of accuracy of the techniques adopted, by GTR itself and, in particular, by $\gamma$ which enters the PPN expressions for the time delay and the bending of traveling electromagnetic waves. Thus, there exists, in principle, the possibility that the high-accuracy results of the Cassini radio science tests may retain an a priori "imprint" of GTR itself through $G M_{\odot}$ (and the Astronomical Unit as well).

Let us put our hypothesis on the test by making some concrete calculations; for the sake of clarity, we will refer to the Cassini radio science tests, but the conclusions may be considered valid also for any of the many proposed $\gamma$-dedicated missions.

The GTR time delay experienced by electromagnetic waves propagating from point 1 to point 2 is

$$
\Delta t=\frac{2 R_{g}}{c} \ln \left(\frac{r_{1}+r_{2}+r_{12}+R_{g}}{r_{1}+r_{2}-r_{12}+R_{g}}\right),
$$

where $R_{g}=2 G M_{\odot} / c^{2}$ is the Sun's Schwarzschild radius in which $G$ is the Newtonian gravitational constant, $M_{\odot}$ is the solar mass, $c$ is the speed of light in vacuum, $r_{1}$ is the heliocentric coordinate distance to point $1, r_{2}$ is the 
heliocentric coordinate distance to point 2 , and $r_{12}$ is the distance between the points 1 and 2. Equation (6) is the expression actually used in the JPL's Orbit Determination Program (ODP) to analyze interplanetary ranging to planets and probes. In order to quantitatively evaluate the level of "imprinting" by GTR itself in the used value of the Astronomical Unit, let us assume that $r_{1}$ equal to the EarthSun distance and let us vary $r_{2}$ within 0.38 au and $1.5 \mathrm{au}$ to account for the ranging to inner planets; the maximum effect occurs at the superior conjunction, that is, when $\mathbf{n}_{1} \approx-\mathbf{n}_{2}$ and $r_{12} \approx r_{1}+r_{2}$. (Here $\mathbf{n} \equiv \mathbf{r} / r$.) It turns out that $\Delta t_{\text {ranging }} \approx 4 \times 10^{-4} \mathrm{~s}$, which is certainly not negligible with respect to the accuracy of the order of $10^{-8} \mathrm{~s}$ with which the light time for 1 au $\tau_{\mathrm{A}}$ is actually measured (http://ssd.jpl.nasa.gov/?constants).(The value in $\mathrm{km}$ of the Astronomical Unit is obtained by measuring at a given epoch the distance between the Earth and a target body (a planet or a probe orbiting it) by multiplying $c$ times the round trip travel time $\tau$ of electromagnetic waves sent from the Earth and reflected back by the target body, and confronting it with the distance, expressed in AU, between the Earth and the target body at the same epoch as predicted by some accurate dynamical ephemeris [14].) As a consequence, the quantitative impact of the interplanetary ranging in the inner solar system on the determination of the Astronomical Unit is of the order

$$
d \mathrm{AU}=c \Delta t_{\text {ranging }}=1.14291 \times 10^{5} \mathrm{~m},
$$

not negligible with respect to the meter-level accuracy in measuring the Astronomical Unit; thus, $d \mathrm{AU} / \mathrm{AU}=8 \times 10^{-7}$. Differentiating (5) with respect to au and (7) yields

$$
\frac{d G M}{G M_{\odot}}=2 \times 10^{-6} .
$$

Thus, we conclude that the technique adopted to determine the numerical values of the Astronomical Unit and of the Sun's $G M$ induced an a priori "imprint" of GTR on them of $8 \times 10^{-7}$ and $2 \times 10^{-6}$, respectively.

Let us apply this result to a typical radio science experiment in the solar system with $r_{1}$ fixed to the EarthSun distance. By writing $r_{1 / 2}=x_{1 / 2}$ au, with $x_{1 / 2}$ expressing distances in Astronomical Units, differentiation of (6) with respect to au and $G M_{\odot}$, and (7)-(8) yields an "imprinting" effect of the order of

$$
\left.\frac{\delta(\Delta t)}{\Delta t}\right|_{\mathrm{GTR}}=2 \times 10^{-6}
$$

for $r_{2}$ up to tens of $\mathrm{AU}$; it turns out that the largest contribution comes from $d G M_{\odot}$. (The Cassini test was performed with $r_{2}=7.43 \mathrm{au}$ [2].) It is too small by one order of magnitude with respect to the performed Cassini radio science tests, but it should be taken into account in the future, more accurate experiments whose expected accuracy is of the order of $10^{-7}-10^{-9}$, in the sense that the a priori bias of GTR in the future determinations of deviations of $\gamma$ from unity will be as large as, or even larger than the effects one will test, unless either $G M_{\odot}$ will be estimated as well along with $\gamma$ itself or a value obtained independently of it will be adopted.

About the first point, we mention that $\gamma$ and $\beta$ were never estimated in those solutions in which, among other things, also AU was fitted; instead, they were kept fixed [1519]. Moreover, Fienga et al. [19] used a modified version of their latest planetary ephemerides, named INPOP08d, in which they kept fixed AU and simultaneously fitted $G M_{\odot}$ to the observations along with other parameters, but they did not simultaneously estimate the PPN parameters as well, which were kept fixed. Instead, for the purposes outlined in this paper it would be important to simultaneously fit $\gamma$ and $\mathrm{AU}$ (or $G M_{\odot}$ ) and inspect the covariance matrix for the correlation among $\mathrm{AU}\left(\right.$ or $G M_{\odot}$ ) and $\gamma$. (Recently, W. M. Folkner (private communication to the author, March 2010) produced a global solution aimed to specifically test general relativity with the latest version of the JPL DE ephemerides. In it $\beta, \gamma$ and $\mathrm{AU}$ were simultaneously fitted obtaining a correlation between $\gamma$ and $\mathrm{AU}$ of $-1.34 \times 10^{-2}$.)

Concerning the last point, a possible choice may consist, in principle, of using a figure for $G M_{\odot}$ obtained from measurements of the solar gravitational redshift

$$
Z \equiv \frac{\nu_{\mathrm{rec}}-\nu_{\mathrm{em}}}{\nu_{\mathrm{rec}}} \approx \frac{G M_{\odot}}{R_{\odot} c^{2}}
$$

which, at first order, is independent of GTR itself. (Indeed, it depends on the coefficient $g_{00}$ of the spacetime metric tensor; only the PPN parameter $\beta$ enters $g_{00}$ with the term of order $\mathcal{O}\left(c^{-4}\right)$, at present, undetectable.) Latest measurements of the IR oxygen triplet 7772-7775, extrapolated to the Sun's limb [20], yield an accuracy of the order of a few percent levels. Projects to improve it with future missions have been proposed [21, 22]; for example, the use of the MagnetoOptical Filter technique, developed by Cacciani et al. [21], would allow reaching a relative accuracy of $10^{-6}$. Anyway, it must be noted that extracting $G M_{\odot}$ from the measured gravitational redshift also requires the knowledge of the Sun's radius $R_{\odot}$, which is uncertain at a $10^{-4}$ level. Indeed, the commonly accepted value for the solar radius was for a long time [23]

$$
R_{\odot}=695.99 \mathrm{Mm}
$$

$\left(1 \mathrm{Mm}=10^{6} \mathrm{~m}\right)$, although it was not clear how such a figure was obtained; moreover, no error bar was released. Subsequent observations of solar $f$-mode frequencies were induced by Schou et al. [24] and Antia [25], who used data from the Michelson Doppler Imager (MDI) mounted on the Solar and Heliospheric Observatory (SOHO) satellite and from the Global Oscillation Network Group (GONG) network; to conclude that the actual solar radius was 0.3-0.2 Mm smaller, indeed, the estimate by Schou et al. [24] is

$$
R_{\odot}=695.68 \pm 0.02 \mathrm{Mm} .
$$

In the work of Brown and Christensen-Dalsgaard [26] the value

$$
R_{\odot}=695.509 \pm 0.026 \mathrm{Mm}
$$


is reported from the Earth-based High Altitude Observatory's Solar Diameter Monitor campaign; it differs by about $0.5 \mathrm{Mm}$ from the standard value by Allen [23]. In the work of Takata and Gough [27] we find, from SOHO/MDI frequency data for $p$-mode frequencies,

$$
R_{\odot}=695.69 \pm 0.14 \mathrm{Mm}
$$

Note that the values by Brown and Christensen-Dalsgaard [26] and Takata and Gough [27] are mutually inconsistent, although at a 1- $\sigma$ level only, the estimates by Schou et al. [24] and Brown and Christensen-Dalsgaard [26] are not consistent at 3- $\sigma$ level. The $f$-mode and $p$-mode measurements $[24,27]$ are, instead, consistent with each other. By the way, it can be noted that the discrepancies among the different best estimates are larger than the associated errors; thus, as a conservative evaluation of the accuracy in knowing the solar radius we will assume such differences.

\section{Conclusions}

We have investigated the impact of possible a priori "imprinting" effects of GTR itself on satellite/spacecraft data analyses specifically designed to test some general relativistic predictions. In particular, we considered the time-delay experiments conducted or proposed in the Sun's field with ranging to interplanetary spacecraft.

Concerning the time-delay tests, the numerical values for the Astronomical Unit and the solar gravitational constant currently adopted in the ephemerides used so far to analyze spacecrafts' data retain an a priori "imprint" of GTR itself of the order of $8 \times 10^{-7}$ and $2 \times 10^{-6}$, respectively. As a consequence, the bias in typical solar system radioscience experiments is of the order of $10^{-6}$, which is one order of magnitude smaller than the accuracy level reached in the performed Cassini experiment, but it would be of concern for future planned tests aiming to measure deviations of the PPN parameter $\gamma$ from its general relativistic value at $10^{-7}-10^{-9}$ level.

In order to have genuine, unambiguous, and unbiased tests of GTR which are not "tautologic", it would be necessary to either estimate the suspect parameters as well along with those accounting for the relativistic effect of interest or use values for them obtained independently from GTR itself.

\section{References}

[1] L. Iess, G. Giampieri, J. D. Anderson, and B. Bertotti, "Doppler measurement of the solar gravitational deflection," Classical and Quantum Gravity, vol. 16, no. 5, pp. 1487-1502, 1999.

[2] B. Bertotti, L. Iess, and P. Tortora, "A test of general relativity using radio links with the Cassini spacecraft," Nature, vol. 425, no. 6956, pp. 374-376, 2003.

[3] J. D. Anderson, E. L. Lau, and G. Giampieri, "Measurement of the PPN parameter $\gamma$ with radio signals from thr Cassini spacecraft at X- and Ka-bands," in Proceedings of the 22nd Texas Symposium on Relativistic Astrophysics, Stanford University, Stanford, Calif, USA, December 2004, eConf C041213, 0305.
[4] W.-T. Ni, "ASTROD and ASTROD I-overview and progress," International Journal of Modern Physics D, vol. 17, no. 7, pp. 921-940, 2008.

[5] N. Ashby, P. Bender, J. L. Hall, et al., "Measurement of the gravitational time delay using drag-free spacecraft and an optical clock," Bulletin of the American Astronomical Society, vol. 41, p. 893, 2009.

[6] D. Hobbs, B. Holl, L. Lindegren, F. Raison, S. Klioner, and A. Butkevich, "Determining PPN gamma with Gaia's astrometric core solution," Bulletin of the American Astronomical Society, vol. 41, p. 891, 2009.

[7] S. G. Turyshev, "Tests of relativistic gravity in space: recent progress and future directions," Bulletin of the American Astronomical Society, vol. 41, p. 887, 2009.

[8] A. Milani, "Relativistic models for the BepiColombo radioscience experiment," Bulletin of the American Astronomical Society, vol. 41, p. 892, 2009.

[9] T. Damour and K. Nordtvedt, "General relativity as a cosmological attractor of tensor-scalar theories," Physical Review Letters, vol. 70, no. 15, pp. 2217-2219, 1993.

[10] T. Damour and K. Nordtvedt, "Tensor-scalar cosmological models and their relaxation toward general relativity," Physical Review D, vol. 48, no. 8, pp. 3436-3450, 1993.

[11] T. Damour and A. M. Polyakov, "The string dilation and a least coupling principle," Nuclear Physics B, vol. 423, pp. 532-558, 1994.

[12] T. Damour, F. Piazza, and G. Veneziano, "Runaway dilaton and equivalence principle violations," Physical Review D, vol. 89, Article ID 081601, 2002.

[13] S. G. Turyshev, "Experimental tests of general relativity," Annual Review of Nuclear and Particle Science, vol. 58, pp. 207248, 2008.

[14] E. M. Standish, "The astronomical unit now, in transits of venus: new views of the solar system and galaxy," in Proceedings of the IAU Colloquium No.196, D. W. Kurz, Ed., pp. 163-179, Lancashire, UK, June 2004.

[15] G. A. Krasinsky and V. A. Brumberg, "Secular increase of astronomical unit from analysis of the major planet motions, and its interpretation," Celestial Mechanics and Dynamical Astronomy, vol. 90, no. 3-4, pp. 267-288, 2004.

[16] E. V. Pitjeva, "High-precision ephemerides of planets-EPM and determination of some astronomical constants," Solar System Research, vol. 39, no. 3, pp. 176-186, 2005.

[17] A. Fienga, H. Manche, J. Laskar, and M. Gastineau, "INPOP06: a new numerical planetary ephemeris," Astronomy and Astrophysics, vol. 477, no. 1, pp. 315-327, 2008.

[18] E. V. Pitjeva and E. M. Standish, "Proposals for the masses of the three largest asteroids, the moon-earth mass ratio and the astronomical unit," Celestial Mechanics and Dynamical Astronomy, vol. 103, no. 4, pp. 365-372, 2009.

[19] A. Fienga, J. Laskar, T. Morley, et al., "INPOP08, a 4-D planetary ephemeris: from asteroid and time-scale computations to ESA mars express and venus express contributions," Astronomy and Astrophysics, vol. 507, no. 3, pp. 1675-1686, 2009.

[20] J. C. Lopresto, C. Schrader, and A. K. Pierce, "Solar gravitational redshift from the infrared oxygen triplet," Astrophysical Journal, vol. 376, no. 2, pp. 757-760, 1991.

[21] A. Cacciani, R. Briguglio, F. Massa, and P. Rapex, "Precise measurement of the solar gravitational red shift," Celestial Mechanics and Dynamical Astronomy, vol. 95, no. 1-4, pp. 425-437, 2006. 
[22] F. Berrilli, M. Velli, L. Roselli, et al., "The ADAHELI solar mission," in Proceedings of the 12th European Solar Physics Meeting, Freiburg, Germany, September 2008.

[23] C. W. Allen, Astrophysical Quantities, Athlone Press, London, UK, 1973.

[24] J. Schou, A. G. Kosovichev, P. R. Goode, and W. A. Dziembowski, "Determination of the Sun's seismic radius from the SOHO michelson doppler imager," Astrophysical Journal, vol. 489, no. 2, pp. L197-L200, 1997.

[25] H. M. Antia, "Estimate of solar radius from f-mode frequencies," Astronomy and Astrophysics, vol. 330, no. 1, pp. 336-340, 1998.

[26] T. M. Brown and J. Christensen-Dalsgaard, "Accurate determination of the solar photospheric radius," The Astrophysical Journal, vol. 500, p. L195, 1998.

[27] M. Takata and D. O. Gough, "The influence of uncertainties in the Sun's radius on inversions for the solar structure," in Proceedings of the SOHO 10/GONG 2000 Workshop: Helioand Asteroseismology at the Dawn of the Millennium, pp. 543546, ESA Publications Division, Santa Cruz de Tenerife, Spain, October 2000. 

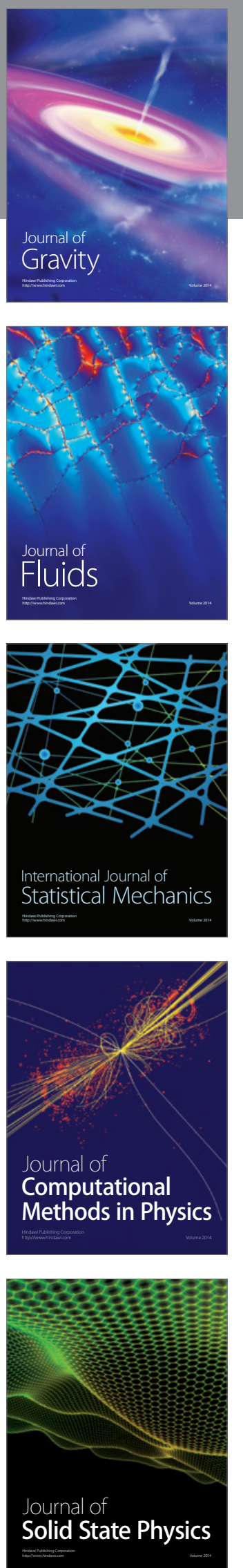

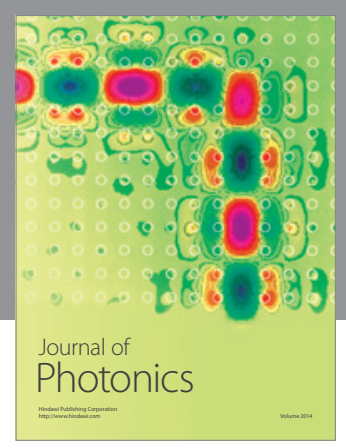

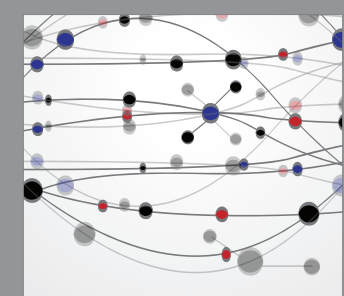

The Scientific World Journal
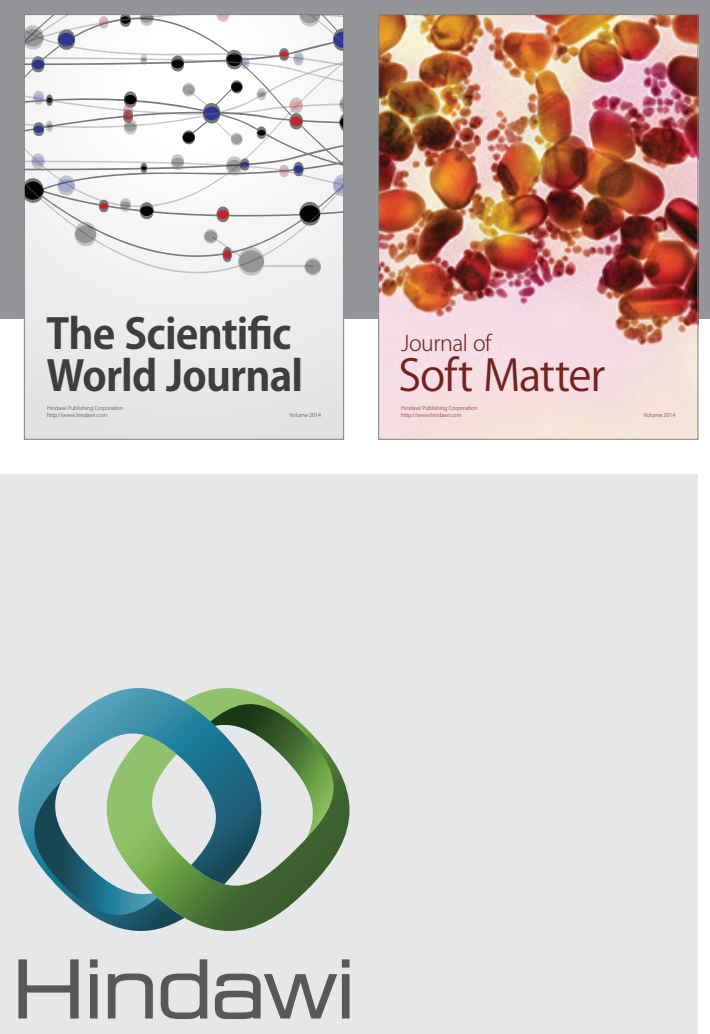

Submit your manuscripts at

http://www.hindawi.com
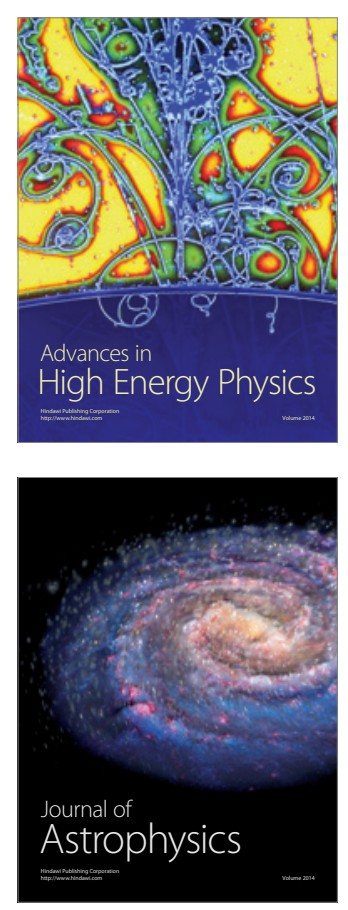
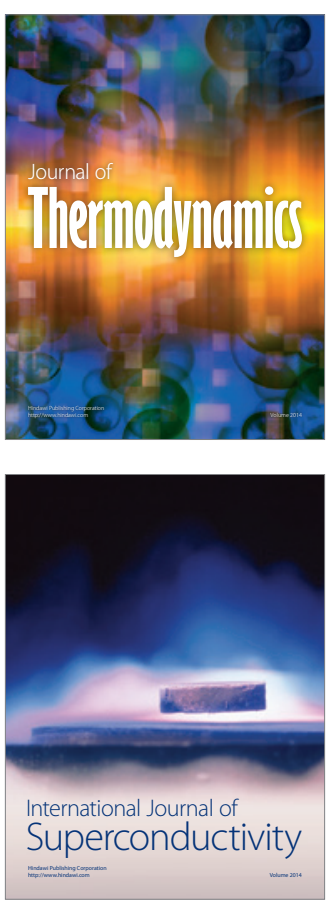
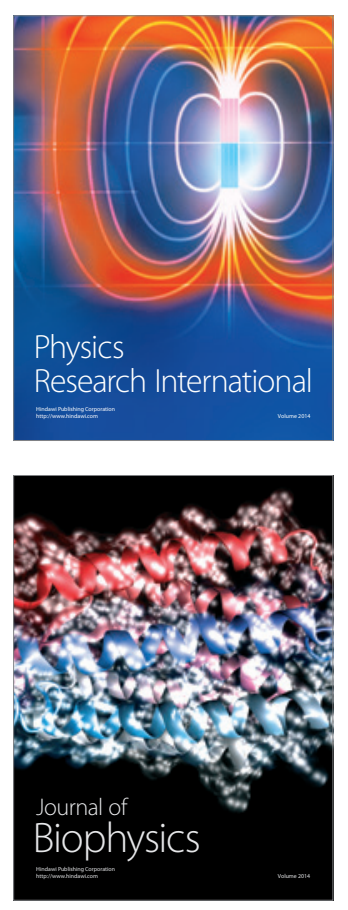
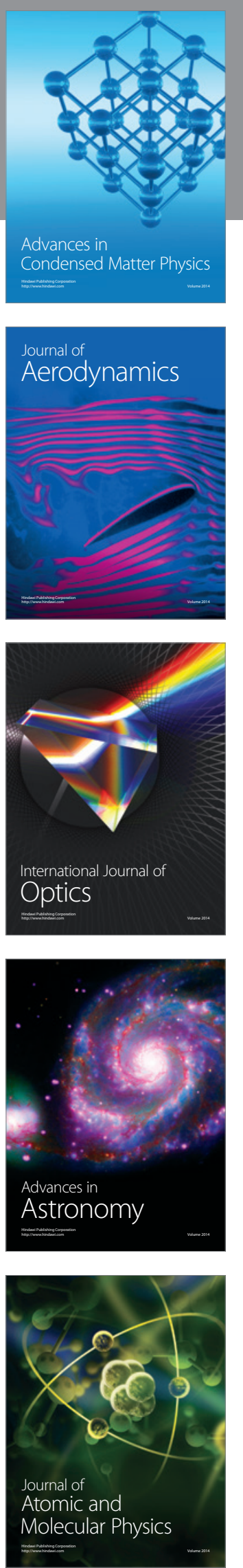